\title{
VolleyJump: Uma aplicação para a análise de saltos no voleibol de praia
}

\author{
Renan Bandeira, \\ Fernando Trinta, \\ João Gomes \\ Universidade Federal do Ceará \\ Av. Humberto Monte, SN - PICI \\ Fortaleza, Ceará \\ renanbandeira@alu.ufc.br,fernando. \\ trinta@dc.ufc.br,jpaulo@lia.ufc.br
}

\author{
Marcio Maia \\ UFC - Campus Quixadá \\ Av. José de Freitas Queiroz, 5003 \\ Quixadá, Ceará \\ marcioefmaia@gmail.com
}

\author{
Alexandre Araripe \\ Universidade Federal do Ceará \\ Av. Humberto Monte, SN - PICI \\ Fortaleza, Ceará \\ alexandremedeiros@ufc.br
}

\begin{abstract}
Professional sports are increasingly dependents of technological resources given the remarkable level of competitiveness faced by high performance athletes. With such resources, it is possible to analyze matches, avoid mistakes that may be committed by the referee or even to analyze the athletes' performance. One of these sports is beach volleyball, one of most popular sports in Brazil. In the past 12 years, the Brazilian volleyball teams has been always among the best teams in the world. The athletes' performance during the jump movement is one of the main factors that one team needs to improve to be successful because it is the movement that is most perfomed during a volleyball match. There are some approaches that study the jump movement in order to calculate its height and give evidences to improve it. Nevertheless, these solutions are expensive and are not viable to athletes with no sponsorship. Having this in mind, this works presents VolleyJump, an application created to analyze beach volleyball athlete jumps using machine learning strategies to calculate the jump height and classify it as an attack or block jump. Results show that VolleyIoT makes possible to analyze athletes' jumps using mobile devices sensors, helping them to focus on their trainning to improve its technique.
\end{abstract}

\section{KEYWORDS}

Wearable Computing, IoT, Mobile Device

\section{INTRODUÇÃO}

Atualmente, o esporte de alto rendimento está cada vez mais dependente de recursos tecnológicos. É difícil pensar em algum esporte em que um aparato tecnológico não esteja por trás de recordes e melhorias de desempenho dos atletas, ou mesmo para que uma comissão julgadora possa avaliar a quebra de recorde, ou analisar os lances durante o jogo e não falhar nos julgamentos realizados. Um destes esportes é o voleibol de praia.

No Voleibol de praia, boa parte das ações básicas de um jogador envolvem saltos. Um atleta salta tanto para ações de ataque à quadra do adversário, quanto na tentativa de bloquear o ataque do oponente.

In: XVII Workshop de Ferramentas e Aplicações (WFA 2018), Salvador, Brasil. Anais do XXIV Simpósio Brasileiro de Sistemas Multimídia e Web: Workshops e Pôsteres.

Porto Alegre: Sociedade Brasileira de Computação, 2018.

(C) 2018 SBC - Sociedade Brasileira de Computação.

ISBN 978-85-7669-435-9.
Um atleta de alto nível neste esporte precisa manter seu nível de saltos de modo a manter seu desempenho homogêneo durante todo o andamento de uma partida. Com isso, analisar os saltos pode gerar resultados muito importantes para a melhoria do desempenho de um atleta. Dentre algumas questões que podem ser obtidas com esta análise estariam: (i) Quantos saltos um atleta dá, em média, durante uma jogo ou treino? (ii) Qual a diferença entre um salto de ataque para um salto de bloqueio? (iii) Como se mantém o desempenho dos saltos do atleta durante o jogo?

A resposta a tais perguntas é essencial para a análise de fadiga do atleta, além de facilitar um treino focado na melhoria de determinados movimentos, e consequente melhoria do desempenho de atletas de voleibol. Nos últimos anos, dispositivos vestíveis tem encontrado um novo nicho no mercado para o monitoramento de performance em esportes como o golfe, futebol, basquete, dentre outros. A partir de dados coletados de sensores em objetos como pulseiras e relógios inteligentes, é possível utilizar técnicas de aprendizado de máquina e técnicas supervisionadas de classificação, para identificar novos dados de saltos a partir de exemplos gerados previamente.

É neste contexto que este documento apresenta a VolleyJump, uma aplicação que se propõe a viabilizar a melhoria de rendimento dos atletas de voleibol de praia a partir do uso de técnicas de aprendizagem de máquina para analisar um treinamento ou jogo e direcionar o treinamento do atleta em deficiências encontradas, de acordo com os resultados da aplicação. Para isso, a aplicação busca coletar dados contextuais do atleta durante o movimento do salto e, a partir destas informações, gerar informações relevantes referentes ao movimento. Com estes dados, o VolleyJump tem como objetivo (a) inferir o momento em que um salto foi realizado, (b) calcular a altura do salto e gerar um modelo de aprendizagem de máquina para que, quando novos dados contextuais de um salto forem gerados, ser capaz de (c) classificá-lo entre salto de ataque ou bloqueio.

\section{TRABALHOS RELACIONADOS}

Identificar a ação de uma pessoa a partir de dados coletados por sensores e dispositivos atrelados ao corpo de um usuário não é algo novo. O tema tem ganhado cada vez mais destaque na área do esporte. [2] e [6] buscam analisar a performance e saúde de atletas durante a prática do futebol e mountain bike, respectivamente. [2] propõe um modelo de arquitetura onde é possível monitorar as condições de saúde do atleta para que seja possível evitar lesões 
futuras. Porém, [6], apresenta um sistema de sensoreamento distribuído a fim de monitorar a performance do atleta de mountain bike com o objetivo de futuras melhorias. No voleibol de quadra, [3] busca identificar saltos realizados durante um jogo analisando a frequência dos dados do acelerômetro.

A análise de saltos no voleibol de quadra é o tema estudado em [7]. Neste trabalho, os autores buscam encontrar alternativas à identificação de um salto de voleibol e o cálculo da altura de maneira rápida, de modo que o atleta possa realizar movimentos durante a prática do esporte e o treinador possa estar ciente do seu desempenho no momento da partida. Para isso, foi desenvolvido um dispositivo vestível que captura os dados do acelerômetro no eixo perpendicular à superfície para que pudesse ser calculado o que o trabalho propõe. Para que o treinador acompanhe o atleta, estes dados gerados são enviados via comunicação de rede sem fio (wireless) ao smart phone, possibilitando que o treinador já tenha a informação gerada a partir do dado segundos depois do realização do salto. A proposta aqui apresentada é semelhante ao proposto por [7], porém com a possibilidade também de se distinguir o tipo do salto do atleta, entre os de bloqueio e de ataque. Esta classificação automática pode ser de grande valia para o treinamento e melhoria do desempenho de um atleta, permitindo que fisiologistas e preparadores físicos possam analisar diferenças acentuadas em relação aos tipos de salto, ou mesmo uma queda de desempenho maior para um tipo de salto em relação a outro durante uma partida. Pelo que foi levantando na revisão de literatura relacionada ao tema, não existem soluções focadas em identificar o tipo de salto, um dos objetivos deste trabalho.

Com o foco em identificar padrões de estratégias bem sucedidas, [8] aplica dados da partida final da copa do mundo de voleibol de quadra de 2014 em técnicas de aprendizagem de máquina a fim de identificar padrões de ataques e defesas bem sucedidas durante o jogo. As estratégias encontradas são relevantes para que a equipe possa vencer o jogo, mas não são direcionadas para o rendimento e recuperação do atleta.

No voleibol de praia, o tema também é estudado. [4] busca criar uma rede neural para identificar atividades realizadas durante a prática do esporte, entre elas os saltos de levantamento e ações relacionadas ao movimento do braço, como o movimento do bloqueio e outros movimentos relacionados ao ataque, com uma acurácia de 83.2\%. No entanto, a altura e os saltos de bloqueio não são levados em consideração.

Entre as técnicas utilizadas na academia, existem diversas delas que utilizam aparatos tecnológicos, em que é possível analisar os saltos de atletas de voleibol. Para medir a altura do salto, existe um dispositivo chamado Vertec, que o atleta salta e deve tocar em parte do aparato, como mostra a figura 1.

Além disso, existem plataformas de força que realizam a análise do salto baseado no tempo de voo do atleta, partindo do momento em que ele já não está mais pisando na plataforma até o momento onde ele volta a encostar nela [5]. A plataforma fump System Optical usa sensores de presença para identificar os momentos de início do salto e queda em solo e a plataforma fump System Pro usa sensores de pressão em sua plataforma para identificar o contato do atleta com o solo e, a partir do tempo de voo, calcular a altura do salto. Ambas plataformas dependem de um solo plano para a

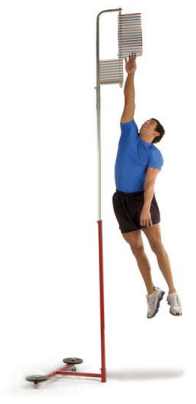

Figura 1: Dispositivo Vertec responsável por medir a altura do atleta. Fonte: roguefitness.com

execução dos saltos, além de que passadas anteriores ao movimento devem ser executados fora da plataforma. Essas abordagens geram um resultado eficiente, mas são soluções caras para o problema proposto.

\section{PROPOSTA}

Para cada salto realizado, esta aplicação propõe identificá-los e mostrar resultados a partir da análise do salto executado. Para isso, o salto deve gerar dados, que devem ser analisados a fim de inferir a altura e o tipo de salto realizado. Portanto, é necessário dois passos para validar esta aplicação: (i) coleta e (ii) análise dos dados. Para o primeiro passo, a aplicação desenvolvida para a plataforma Android chamada VolleyJump permite a coleta de dados de sensores portados por um atleta, a fim de inferir dados sobre saltos. Os dados são enviados para um serviço de banco de dados da nuvem, responsável por armazenar os dados de maneira em que seja possível acessá-los a partir de qualquer dispositivo com acesso a Internet a qualquer momento. Com esses dados, um servidor na nuvem busca aplicar técnicas de aprendizagem de máquina e identificar o tipo e altura dos saltos, enviando essa informação ao dispositivo. A figura 2 apresenta este fluxo de troca de dados.

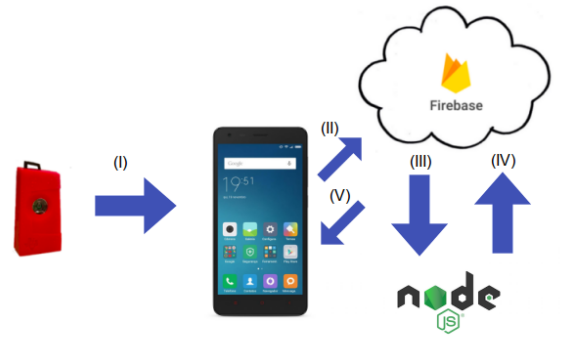

Figura 2: Fluxo de comunicação e troca de dados no VolleyJump

No desenvolvimento da VolleyJump, os dados são coletados pelo sensor e repassados ao smarthphone (passo 1) e enviados a um banco de dados na nuvem, o Firebase (passo II). O servidor é notificado (passo III) e analisa os dados, retornando o resultado da análise 
para Firebase (Passo IV). Por fim, a aplicação móvel é notificado ao final da análise (Passo V).

No caso desta aplicação, o diferencial em relação aos trabalhos relacionados é a categorização dos saltos em ataque ou bloqueio, utilizando assim técnicas de aprendizagem automática supervisionado de classificação. Este trabalho propõe o uso de uma estratégia de aprendizagem de máquina supervisionado, pois durante a execução do salto, é possível identificar o tipo e enviar ao algoritmo para que ele aprenda a partir de exemplos. Inicialmente, estes exemplos devem vir com o tipo e altura do salto, além dos dados gerados pelos sensores. Estes exemplos são essenciais para que a aplicação possa gerar um modelo de aprendizagem de máquina e, a partir de novos saltos, a aplicação possa inferir o tipo e a altura do salto. Para gerar estes exemplos, um grupo de possíveis atletas realizou saltos em uma plataforma usando o dispositivo que captura dados sensoriais a fim de que todos os dados necessários para a geração do modelo de aprendizagem de máquina usando o algoritmo de máquinas de vetor de suporte. Após gerar o modelo, novos saltos foram realizados sem o auxílio da plataforma de força para que a própria aplicação identifique e calcule a altura e o tipo de salto. Finalmente, os resultados são comparados.

\section{VISÃO GERAL}

Para que a aplicação possa contemplar o que este trabalho propõe, foi necessária a criação de duas telas: a primeira para a coleta dos dados e a segunda para mostrar os resultados obtidos, como mostra a Figura 3.

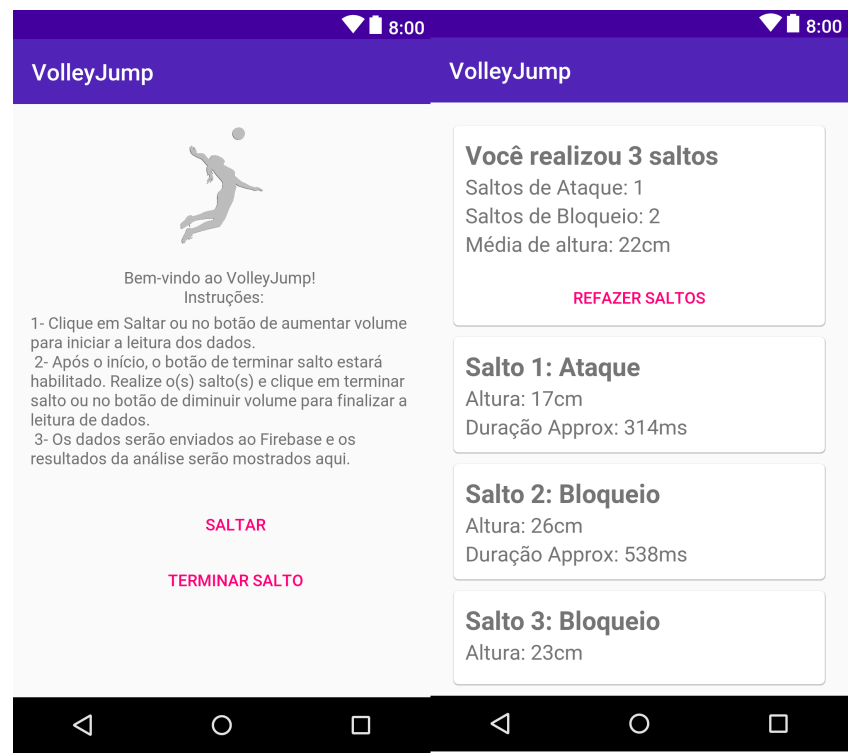

Figura 3: VolleyJump: tela responsável pela coleta de dados no lado esquerdo e os resultados no lado direito

\subsection{Coleta de Dados}

A fim de deixar o atleta confortável durante a prática do esporte, um dispositivo vestível é acoplado ao corpo do atleta e se comunica com a aplicação Android a fim de enviar os dados coletados. Essa comunicação usa o padrão de projetos Observer [1] para o envio de dados gerados por sensores e, para isso, é preciso que o VolleyJump registre um SensorTagListener, que é uma interface que contém o método onSensorTagUpdate e passa como parâmetro um objeto do tipo String com os dados do sensor. Essa String é um JSON (do inglês, Javascript Object Notation).

Como o foco desta aplicação é o movimento do salto, a identificação do salto por acelerômetro é o objetivo do uso do sensor de acelerômetro acoplado ao usuário para descobrir os valores de aceleração nos 3 eixos dimensionais. A partir do padrão de mudança de aceleração durante o movimento do salto, é possível identificar saltos analisando apenas o valor da aceleração do eixo perpendicular à superfície, ou seja, o eixo Y.

\section{EXPERIMENTOS REALIZADOS}

Para verificar a viabilidade da proposta do VolleyJump, foram realizados testes com um protótipo desenvolvido de acordo com a arquitetura proposta. As tecnologias utilizadas foram: (i) plataforma Android para a aplicação Móvel, (ii) Node.js para o serviço de inferência de saltos, (iii) banco de dados Firebase e (iv) comunicação via Bluetooth LE. Esta seção descreve os experimentos realizados e os resultados obtidos. Como o trabalho também impõe que a coleta dos dados de saltos de um atleta de voleibol de praia a fim de que esta coleta seja feita de maneira imperceptível sem que gere um desconforto ao atleta, foi inicialmente utilizada o SensorTag CC26505 da Texas Instruments, usando uma cinta para acoplá-lo ao peito do atleta, como mostra a figura 4 .

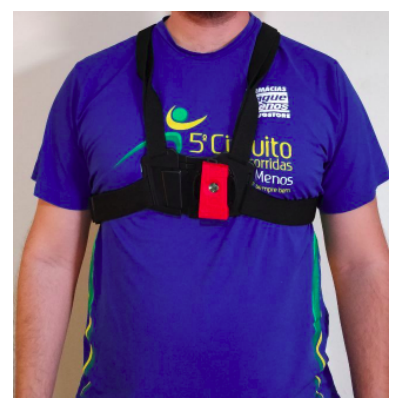

Figura 4: Sensortag é acoplado ao peito do atleta a fim de coletar dados durante o salto

Na fase inicial da criação do VolleyJump, para fins de estudo do modelo, foram realizados trinta saltos, entre saltos de ataque e bloqueio, usando a SensorTag CC26505. Estes saltos foram coletados no Instituto de Educação Física da Universidade Federal do Ceará (IEFUFC), acompanhados por um professor de educação física especializado no treinamento de alto rendimento para atletas de voleibol de praia. Durante a sessão de saltos, os movimentos foram realizados também sobre plataformas de força disponíveis no instituto capazes de informar a altura dos saltos realizados, entre elas a fump System Duo ${ }^{1}$, e a Jump System Optical ${ }^{2}$, como mostra a figura 5. A amostragem dos valores coletados pelo sensor foi configurada para alta acurácia, a maior possível. Esta decisão segue a orientação de se

\footnotetext{
${ }^{1}$ http://www.cefise.com.br/produto/104/30/jump-system-duo

${ }^{2}$ http://www.cefise.com.br/produto/78/30/jump-system-optical
} 
trabalhar com o maior número de amostras possíveis dos sensores de modo a obter maior acurácia da inferência, quando da aplicação dos algoritmos de classificação.

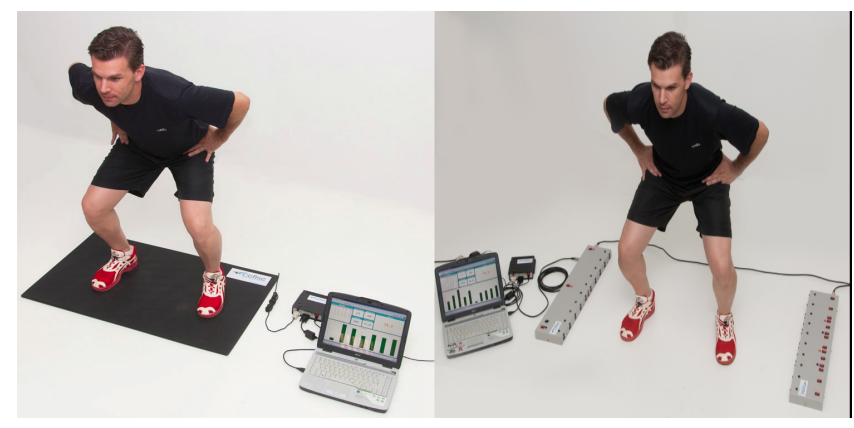

Figura 5: Plataformas utilizadas para validação do trabalho

Durante a análise destes dados, notou-se que os dados gerados pelo dispositivo, ainda que com uma frequência alta de captura de dados, não estavam de acordo com o padrão de dados gerados em um salto como foi mencionado em [7]. A partir de vários experimentos, verificou-se que a SensorTag CC26505 utilizada em nossos experimentos era muito sensível a variações de seu posicionamento na cinta do atleta. Devido a indisponibilidade de outra tag, optou-se por usar o próprio smart phone Android acoplado ao peito usando uma cinta como forma melhorar a qualidade dos dados obtidos, uma que de acordo com a arquitetura proposta para o VolleyJump, a aquisição dos dados pode ser feita a partir de qualquer dispositivo/sensor capaz de obter dados de um acelerômetro e giroscópio, presos ao corpo do atleta. Os smart phone Android utilizados foram o Motorola Moto E, Lenovo Vibe K5 e OnePlus 5T. Nesta nova configuração, foram coletados sessenta saltos de um voluntário, divididos entre trinta saltos de ataque e trinta de bloqueio, usando os sensores do celular na altura peito. Estes experimentos tem como objetivo gerar um modelo de classificação do salto, para que ele pudesse ser validado posteriormente. Portanto, cada salto era realizado de maneira discreta, ou seja, o atleta comunicava ao VolleyJump o momento em que cada salto era realizado, gerando dados separados por salto.

Com o modelo de classificação gerado, uma nova etapa de coleta de dados foi realizada a fim de validar este modelo e repetir o treinamento. Este experimento foi feito com três estudantes voluntários do curso de Educação Física da Universidade Federal do Ceará, sendo 2 do sexo feminino e 1 do sexo masculino, onde realizou-se uma quantidade total de 127 saltos usando o VolleyJump e as plataformas de força mencionadas anteriormente. Estes voluntários representam uma faixa etária de entre 21 e 25 anos, com altura entre $1,80 \mathrm{~m}$ e $1,85 \mathrm{~m}$, pesando entre $80 \mathrm{~kg}$ e $85 \mathrm{~kg}$. Os voluntários não alegaram que o sensor pudesse atrapalhar o desempenho dos seus saltos nem sentiram desconforto ao realizar o salto usando o sensor acoplado ao peito. Os saltos foram realizados usando um protocolo de resistência definido com auxílio do professor de Educação Física que auxilia esta pesquisa, onde o atleta deve saltar o máximo que pode em 5 minutos, com intervalos de 5 segundos entre os saltos. Nesses 5 segundos, o atleta apenas se preparava para realizar o salto seguinte na plataforma, sem que houvesse uma intervenção para que o VolleyJump pudesse identificar este salto, ou seja, os dados coletados neste conjunto de saltos era contínuo, fazendo com que o VolleyJump identificasse os saltos. A partir deste experimento, foi possível validar o VolleyJump quanto a identificação, cálculo de altura e classificação dos saltos.

\subsection{Identificação do Salto}

A fim de identificar os saltos realizados, o algoritmo buscava o padrão de aceleração do corpo no decorrer do tempo referente aos saltos mencionado em [7] a cada segundo, pois nos experimentos iniciais notou-se que os saltos teriam uma duração média de 1 segundo, divididos entre tempo no ar e tempo no solo. Com isso, dos 127 saltos realizados no experimento, o algoritmo identificou 129 saltos, gerando um erro de $1,5 \%$ de falso positivo.

Percebe-se que o processo de classificação a cálculo de altura do salto depende totalmente da identificação do salto, pois a partir dos saltos identificados que o VolleyJump irá classificar e calcular a altura dos saltos realizados, uma vez que, ao identificar o salto, é possível obter o tempo de duração do salto, além dos dados do acelerômetro, gravide e giroscópio neste intervalo.

\subsection{Altura dos Saltos}

Em relação à estimativa da altura dos saltos, os valores estimados pelo VolleyJump foram comparados com aqueles obtidos pelas plataformas de força. Nas plataformas de força, os saltos tinham 15,27 centímetros de altura em média, enquanto que o VolleyJump gerou resultados de 14,06 centímetros, tendo um erro absoluto médio de 1,30 centímetros em relação às plataformas utilizadas. As plataformas de salto são sensíves ao contato do voluntário com o chão, e isso fez com que 14 dos 127 saltos fossem ignorados dos cálculos, pois a plataforma não mostrava dados reais.

\subsection{Classificação dos Saltos}

Após a coleta dos 60 saltos realizados no primeiro experimento válido, o algoritmo gerou um modelo de classificação destes saltos usando um algoritmo de máquinas de vetores de suporte, a fim de que, no momento da coleta dos dados, não fosse mais necessário que o jogador classifique os saltos sempre que a ação for executada. Para a geração do modelo, os dados foram ordenados de maneira aleatória e separados entre dados de treino e validação do modelo, onde $70 \%$ dos dados foram usados para treinar o modelo e os $30 \%$ restantes foram usados para a validação. Para evitar que o modelo ficasse tendencioso, os saltos separados para a realização do treino do modelo estavam divididos igualmente entre os saltos de ataque e bloqueio.

A figura 6 ilustra o padrão dos dados do acelerômetro e giroscópio no eixo Y na realização de uma sequência de 5 saltos. É possível perceber o padrão da identificação do salto caracterizado pela queda brusca de aceleração para tomar impulso e recuperação da aceleração ao finalizar o salto. No giroscópio, é importante perceber o intervalo de valores resultantes durante o salto, variando entre -0.9 e 0.9 .

A fim de comparar os saltos, a figura 7 ilustra os dados coletados durante 6 saltos. O padrão de identificação do salto permanece ao analisar os dados do acelerômetro e o giroscópio tende a ser mais variável, variando entre aproximadamente 2 e -2 . Essa maior 


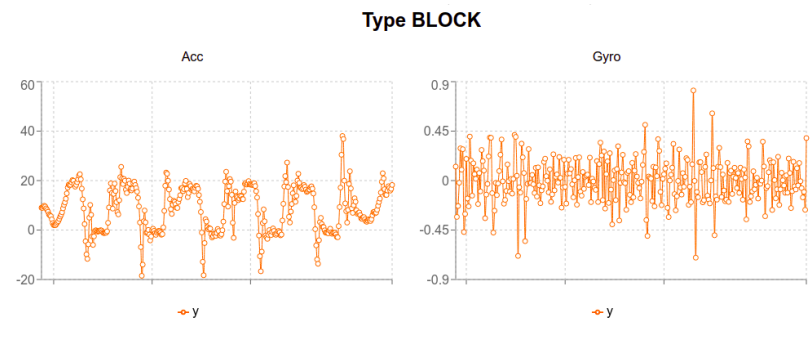

Figura 6: Acelerômetro e giroscópio no eixo Y no momento de uma sequência de saltos de bloqueios

variância do giroscópio no eixo Y ocorre porque o salto de ataque é caracterizado por uma por um avanço prévio ao salto, realizando uma passada antes do salto, diferente do salto de boqueio, onde o atleta salta praticamente partindo do mesmo lugar em que se encontra. Os dados coletados nos eixos X e Z não representaram um padrão que pudesse ser relevante às diferenças de ataque entre salto e bloqueio.

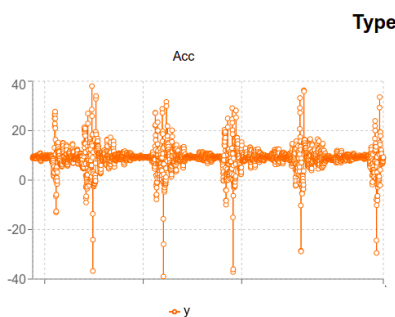

Type ATTACK

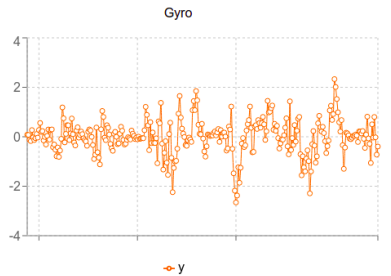

Figura 7: Acelerômetro e giroscópio no eixo Y no momento de uma sequência de saltos de ataque

No último experimento realizado, um conjunto de 85 saltos de bloqueio e 42 saltos de ataque foram executados. A tabela 1 resume os resultados da classificação dos saltos a partir destes saltos.

Tabela 1: Dados coletados durante experimentos com voluntários

\begin{tabular}{|l|c|c|}
\hline & Classif. como Bloqueio & Classif. como Ataque \\
\hline Bloqueio & 78 & 5 \\
\hline Ataque & 12 & 32 \\
\hline Total & 90 & 37 \\
\hline
\end{tabular}

Na validação do modelo, obteve-se uma taxa de acerto de 86,61\% a partir do experimento realizado, onde $93,97 \%$ dos saltos de bloqueio e $72,72 \%$ dos saltos de ataque foram corretamente classificados. $\mathrm{O}$ resultado apresentado é promissor, porém observa-se a necessidade de ajustar o modelo pra que reduza o numero de saltos de ataques classificados erroneamente como saltos de bloqueio. Uma possível explicação para este fenômeno está na menor quantidade de saltos de ataque utilizados para construir o modelo de classificação. O desbalanceamento entre as classes (saltos de ataque e saltos de bloqueio) tende a construir modelos que privilegiam a classe majoritária (com mais exemplos). Desta forma, a continuação deste trabalho prevê uma maior coleta de dados de ataque para reduzir o impacto do desbalanceamento no desempenho do modelo.

\section{CONCLUSÃO}

O uso de tecnologias digitais é uma tendência cada vez maior em esporte de alto rendimento. Este trabalho apresentou como técnicas de aprendizagem de máquina podem ser utilizadas para classificar saltos de voleibol de praia, e também inferir a altura destes saltos a partir de dados de acelerômetro gravidade e giroscópio presos ao corpo de um atleta. Este trabalho apresenta um aplicativo chamado Volleyfump, desenvolvido para a plataforma Android, que apresentase como uma alternativa mais econômica a análise do desempenho dos atletas de voleibol de praia.

O VolleyJump mostrou que é viável realizar análises de movimentos de atleta sem um alto custo financeiro. A aplicação pretende ser distribuída na forma de software livre sobre a licença Apache 2.0, a fim de que estudantes, profissionais da Educação Física e equipes de voleibol de praia amadoras e profissionais possam ter acesso a uma ferramenta de baixo custo com resultados que possam viabilizar a melhoria de rendimento entre atletas. Como trabalho futuro a fim de aumentar o conforto do atleta, deseja-se criar um protótipo de um sensor vestível ao atleta sem que seja necessário acoplar um dispositivo móvel ao seu corpo. O código-fonte do VolleyJump pode ser visto em https://github.com/renanbandeira/VolleyJump.

\section{REFERÊNCIAS}

[1] Ralph Johnson Richard Helm Erich Gamma, John Vlissides. 1994. Design Patterns: Elements of Reusable Object-Oriented Software.

[2] Mohammed Abdulaziz Ikram, Mohammad Dahman Alshehri, and Farookh Khadeer Hussain. 2015. Architecture of an IoT-based system for football supervision (IoT Football). 2015 IEEE 2nd World Forum on Internet of Things (WF-IoT) (2015), 69-74.

[3] Jon M Jarning, Kam-Ming Mok, Bjoerge Herman Hansen, and Roald Bahr. 2015. Application of a tri-axial accelerometer to estimate jump frequency in volleyball. Sports biomechanics 141 (2015), 95-105.

[4] Thomas Kautz, Benjamin H. Groh, Julius Hannink, Ulf Jensen, Holger Strubberg, and Bjoern M. Eskofier. 2017. Activity recognition in beach volleyball using a Deep Convolutional Neural Network. Data Mining and Knowledge Discovery 31, 6 (01 Nov 2017), 1678-1705.

[5] Nicholas P. Linthorne. 2001. Analysis of standing vertical jumps using a force platform. American fournal of Physics 69 (2001).

[6] Tiago Ribeiro, Octavian Postolache, and Pedro Passos. 2016. Performance assessment for mountain bike based on WSN and cloud technologies. 2016 International Conference and Exposition on Electrical and Power Engineering (EPE) (2016), 380386.

[7] Lilian K. S. Sunna. 2010. Athlete monitoring via wireless sensor on a mobile phone. Master's thesis.

[8] Jan Van Haaren, Horesh Ben Shitrit, Jesse Davis, and Pascal Fua. 2016. Analyzing Volleyball Match Data from the 2014 World Championships Using Machine Learning Techniques. Conference: Conference: the 22nd ACM SIGKDD International Conference (08 2016), 627-634 